\title{
Die Neue Übergangsverfassung der Republik Südafrika Ende der Apartheid - Aufbruch in die Demokratie
}

\author{
Von Hans Friedrich Heese und Thomas H. Böhnke
}

Nach einer hitzigen Wahlkampf zeit, die immer wieder von Gewalttaten überschattet war, fanden in Südafrika vom 26. - 29. April 1994 die ersten allgemeinen Wahlen statt. Zeitgleich mit dem zweiten Tag $^{1}$ dieser Wahlen trat auch die Neue Interimsverfassung ${ }^{2}$ der Republik Südafrika am 27. April 1994 in $\mathrm{Kraft}^{3}$ und leitete so eine neue Ära ein, die die Folgen der Apartheidspolitik der vergangenen Jahrzehnte überwinden und zu einem wirklichen Miteinander der verschiedenen Rassen, Sprachgruppen, Stämme, Religionen und Kulturen führen soll.

Dieser Übergang des alten, von der Völkergemeinschaft isolierten, Südafrikas der Apartheid in ein neues Südafrika wurde landesweit unter großer Beteiligung des Volkes als feierliches Ereignis zelebriert, indem kurz vor Mitternacht des 26. April 1994 die alte südafrikanische Staatsflagge eingeholt und einige Minuten nach Anbruch des 27. April die neue südafrikanische Staatsflagge unter den Klängen der neuen Nationalhymnen ${ }^{4}$ gehißt wurde.

$\mathrm{Da}$ dieser Wandel tatsächlich stattfinden würde, war aber lange Zeit keineswegs gewiß. Insbesondere der, bis kurz vor dem Wahltermin aufrechterhaltene, Wahlboykott der Inkatha Freiheitspartei Party ${ }^{5}$ und die vielerorts immer wieder ausbrechende Gewalt verhießen nichts Gutes. Insofern war es keineswegs überraschend, des öfteren Stimmen zu

1 Der Vortag diente der Stimmabgabe durch special voters (Schwangere, kranke und gebrechliche Menschen, vom Wohnort auswärtig stationierte Armeeangehörige, Polizisten und Angehörige der National Peacekeeper, im Ausland lebende Südafrikaner in den dort errichteten Stimmlokalen) nach $\S \S 39$ und 40 des Electoral Act 1993 Act no. 202 of 1993 i.V.m. §§ 36-40 der Electoral Regulation No. R. 310 vom 17.2.1994.

Constitution of the Republic of South Africa, Act No. 200 of 1993; Constitution of the Republic of South Africa Amendment Act, Act No.2 of 1994; Constitution of the Republic of South Africa Second Amendment Act - Act No. 3 of 1994.

3 Art. 251 der Verfassung; abgedruckt im Anhang

4 "Die Stem" und "Nkosi Sikelel'iAfrika".

5 Siehe dazu Karpen, Ulrich in der FAZ vom 17.5.94 S. 10. 
hören, die befürchteten, daß die Wahlen in einer Welle der Gewalt untergehen könnten und Südafrika so einer Zeit des Chaos gegenüberstehen würde. ${ }^{6}$

Doch das fast Unmögliche geschah. Quasi in letzter Minute wurde zwischen der Regierung, dem ANC und der IFP am 19. April eine Übereinkunft erzielt, in der die IFP die Teilnahme an den Wahlen erklärte und versicherte, für einen freien und fairen Ablauf der Wahlen einzutreten, und Regierung und ANC im Gegenzug dazu einer Verfassungsergänzung $^{7}$ zustimmten, die es der Provinz KwaZulu/Natal erlauben wird die Rolle, Autorität und Stellung eines traditionellen Monarchen - eben des Königs der Zulus - in einer Provinzverfassung zu regeln. Und als der Wahltag nahte, hörte sogar die Gewalt auf. Selbst in den Townships, die bis dahin durch tägliche Kämpfe zwischen rivalisierenden politischen Gruppen für traurige Schlagzeilen gesorgt hatten, standen dieselben Menschen, die noch kurz vorher gegeneinander gekämpft hatten gemeinsam in langen Schlangen vor den Wahllokalen, um ihre Stimme abzugeben.

Diese unter der aufmerksamen Beobachtung der Weltöffentlichkeit ${ }^{8}$ durchgeführten Wahlen waren von organisatorischen Problemen in der Durchführung überschattet, die nur durch die große Disziplin und den bewundernswerten Gleichmut der Wähler ausgeglichen werden konnten. Fast überall warteten die Menschen in kilometerlangen Schlangen vor den Wahllokalen. Manche Menschen hatten weite Wege zu Fuß zurückgelegt und mußten dann feststellen, daß dieses Wahllokal noch nicht geöffnet war, weil es an Stimmzetteln, Wahlurnen oder IFP-Aufklebern ${ }^{9}$ fehlte. Und die Menschen warteten friedlich, manchmal sogar über Nacht, nur um ihre Stimme abzugeben. Mit diesen Bildern von friedlich miteinander wartenden Menschen, die von den Medien in alle Welt getragen wurden, demonstrierte Südafrika auf eindrucksvolle Weise den Willen seiner Menschen, einen friedvollen Neuanfang auf eine gemeinsame Zukunft hin zu machen.

Erwartungsgemäß erhielt der ANC südafrikaweit mit 62,65 Prozent eine deutliche Mehrheit gegenüber der Nationalen Partei mit 20,39 Prozent und der Inkatha-Freiheitspartei mit 10,54 Prozent. ${ }^{10}$ In den Provinzen konnte die Nationale Partei mit 53,3 Prozent die

Viele wohlhabende Südaf rikaner hatten über die Zeit der Wahlen das Land verlassen, um aus sicherer Entfernung die Entwicklung abzuwarten. die IFP bedruckt worden. Daher mußte jeder Stimmzettel um einen IFP-Aufkleber am unteren Stimmzettelrand ergänzt werden.

Außer den Genannten zogen die Freiheitsfront mit 2,17\%, die Demokratische Partei mit 1,73\%, der Panafrikanische Congress mit 1,25\% und dieAfrikanische Christlich-Demokratische Partei mit 0,45\% in die Nationalversammlung ein. 
Mehrheit in der Western Cape Provinz und die Inkatha mit 50,3 Prozent in der Provinz KwaZulu/Natal erringen.

Aufgrund dieses Wandels wurde Südafrika eine neue Akzeptanz zu teil, die sich eindrucksvoll bei der Amtseinführung von Nelson Mandela als erstem schwarzen Präsidenten Südafrikas zeigte, indem sich zu diesem Ereignis am 10. Mai 1994 neben dem UN-Generalsekretär die Staatsoberhäupter, Regierungschefs und Außenminister aus mehr als 160 Staaten im Amphitheater des Union Buildings in Pretoria versammelt hatten. Sehr schnell erfolgte dann die Integration in die Völkergemeinschaft; so wurden die letzten Sanktionen gegen Südafrika am 24. Mai 1994 vom Sicherheitsrat der Vereinten Nationen aufgehoben; Südafrika wurde am 25. Mai das 53. Mitglied in der Organisation Afrikanischer Einheit (OAU), 109. Mitglied in der Bewegung der Blockfreien (31. Mai) und 51. Mitglied bei den im Commonwealth zusammengeschlossenen Ländern (1. Juni). Darüber hinaus wurde Südafrika finanzielle Hilfe zur Überwindung der Apartheid und zur Lösung der vielfältigen Aufgaben zugesagt ${ }^{11}$ und mehrere Handelsabkommen zum Ausbau und zur Intensivierung der Wirtschaf tsbeziehungen geschlossen. ${ }^{12}$

\section{Historische Verfassungsentwicklung}

Als 1652 die europäische Siedlung am Kap gegründet wurde, war sie Teil der privaten Handelsgesellschaft der Verenigde Oost-Indische Compagnie (VOC) ${ }^{13}$, die bereits 1602 einen Freibrief der Generalstaaten - dem höchsten Gesetzgebungsorgan der Vereinigten Republik der Niederlande - erhalten hatte, der die Erwerbung überseeischer Besitzungen erlaubte und auf diese das Holländisch-Römische Recht erstreckte. ${ }^{14}$

Diese am Kap entstandene Siedlung wurde durch einen Politischen Rat regiert, in dem alle legislative, exekutive und auch judikative Gewalt ruhte, und der aus dem Kommandanten (dem späteren Gouverneur) und Beamten der VOC gebildet wurde. In der ganzen Zeit unter dem Einfluß der VOC bis 1795, hatte kein ziviler Einwohner des Kaps irgendeine Vertretung in dieser Regierung. Zivilisten (Bürger) spielten lediglich in der Kommu-

Beispielsweise wurden zur Unterstützung des Rekonstruktions- und Entwicklungsplans der Regierung \$ 600 Mio durch die USA - verteilt auf drei Jahre, 107 Mio. Rand und zusätzliche Projektunterstützung durch die Bundesrepublik für 1994, 200 Mio. Rand durch die Schweiz und \$ 1,3 Mill. durch Japan zugesagt.

12 So unter anderem mit China, Indien, Großbritanien, USA, Simbabwe, - eine besondere Regelung mit der EG scheiterte vorläufig am Widerstand Frankreichs.

13 Marais, D., Constitutional Development of South Africa, Johannesburg 1985, S. 4. Das umfassendste und aktuellste Geschichtswerk mit Blick auf die verfassungsgeschichtliche Entwicklung ist das Werk von Fisch, J., Geschichte Südaf rikas, München 1990.

14 Vgl. Muller, C.F.J. (Hrsg.), 500 Jaar Suid-Afrikaanse Geskiedenis, Pretoria und Kapstadt 1968, S. 18; siehe auch Kruger, D.W. (Hrsg.), Geskiedenis van Suid-Afrika, Kapstadt n.d., S. 32, 42. 
nalverwaltung eine geringfügige Rolle, nachdem sich die Siedlung ins Landesinnere ausgedehnt hatte und die VOC es zu teuer fand, Löhne für eine gebührende Verwaltung zu zahlen.

Die insofern getroffene Form der Verwaltung, das System der Landdrost und Heemraden, ${ }^{15}$ bestand aus bezahlten Verwaltern, die von unbezahlten Mitgliedern des Volkes, die durch den Politischen Rat ernannt waren, unterstützt wurden. Ihre Aufgabe bestand vor allem darin, auf kommunale Verwaltungsangelegenheiten zu achten, wie z.B. die Instandhaltung der Straßen und die Führung der Listen von wehrfähigen Männern.

Von 1657 an, als es die ersten Freien Bürger gab (Menschen, die nicht Vollzeitbeschäftigte der VOC waren), wurden Bürgerstadträte ernannt, um die freie weiße Bevölkerung zu repräsentieren. Diese Bürgerversammlungen waren aber ohne Macht; sie wurden lediglich vom Gouverneur hinzugezogen, um Fragen, die die Zivilbevölkerung betrafen, zu beraten. Ihr reale Funktion bestand vielmehr darin, die Bevölkerung über die Entscheidungen des Gouverneurs zu informieren.

1795 besetzten die Briten das Kap. Unter der Regierung eines britischen Militärgouverneurs blieb die rechtliche und politische Situation überwiegend unverändert. Die Rebellionen, die sich in Swellendam und Graaff-Reinet ereigneten, wo "auf sässige" Bürger ihre Selbstbestimmung proklamierten, wurden einfach zerschlagen. Der Britische Gouverneur hatte keinerlei Interesse, den "Holländischen Subjekten mit ihren Jakobinischen Sympathien" politische Rechte zu gewähren. ${ }^{16}$ Im Jahre 1797 erhielt das Kap den Status einer Kronkolonie, und nur einige kleine Veränderungen fanden statt. Die Bürgerversammlung wurde durch einen Bürgersenat ersetzt, dessen Mitglieder ebenfalls ernannt und nicht gewählt wurden. Immerhin hatte dieses neue Organ rudimentäre Kommunalverwaltungskompetenzen. ${ }^{17}$

Dem Vertrag von Amiens entsprechend, wurde das Kap an die Holländische Republik zurückgegeben. Ungeachtet der Zeit der Aufklärung in Europa veränderte sich durch die erneute holländische Herrschaft weder die politische Situation der Bewohner am Kap, noch fanden die Gedanken der Französischen Revolution Einfluß in die Gesetzgebung. In Kapstadt ersetzte zwar ein Gemeinderat ("Raad der Gemeente") aus 7 Stadtbewohnern den Bürgersenat. ${ }^{18}$ Aber auch diese neue Institution hatte nur begrenzte Kommunalgewalt.

Davenport, T.R.H., South Africa. A Modern History, Johannesburg 1980, S 18.

Davenport, T.R.H., South Africa. A Modern History, S. 29.

Marais, D., Constitutional Development of South Africa, S. 10.

Muller, C.F.J., (Hrsg.), 500 Jaar Suid-Afrikaanse Geskiedenis, S. 93. 
1806 wurde das Kap erneut von den Briten erobert. Obwohl beschlossen war, daß das Territorium nach einem formellen Friedensvertrag wieder den Holländern zurückgegeben werden sollte, bestimmte der Londoner Vertrag von 1814, daß das Kap eine Britische Kolonie wurde. Sie wurde durch einen autokratischen Gouverneur regiert, der ein Regierungssystem errichtete, das den örtlichen Einwohnern keinerlei Einflußmöglichkeiten ließ, waren sie nun Holländer oder Eingeborene ${ }^{19}$; der Mangel an politischen Rechten am Kap wurde nur noch durch die Sträflingskolonie New South Wales übertroffen. ${ }^{20}$

Mit dem Jahre 1820 setzte ein reger Zustrom britischer Siedler ein. Diese neuen Einwanderer traten in der neu errichteten Presse - eine solche fehlte in der Holländischen Periode - lebhaft für ihre politischen Rechte ein. Von da an ging der Kampf um politische Rechte Hand in Hand mit dem Ausbau der freien Presse. Aufgrund dieser immer stärker erhobenen Forderung nach politischen Rechten wurde 1825 ein Advisory Council eingeführt, womit die Ära der Ein-Mann-Regierung oder der Regierung durch Bekanntmachung zu Ende ging. ${ }^{21}$ Dieser Council bestand zunächst nur aus Beamten, die den Gouverneur berieten und wurde 1827 um zwei Kolonisten ergänzt. Diese zwei waren jedoch Beamte im Ruhestand, so daß auch sie nicht die niedergelassenen Siedler repräsentierten. ${ }^{22}$

Für die eingeborenen Menschen der Kolonie war das Jahr 1828 von besonderer Bedeutung, da durch die Verkündung der Ordinance 50 alle Beschränkungen, die für die Khoikhoi-People galten, aufgehoben und die Eingeborenen den weißen Einwohnern gleichgestellt wurden. Unter den Bestimmungen der Ordinance 49 wurde Schwarzen - sie wurden bis zu diesem Zeitpunkt nicht als Britische Untertanen unter Kolonialkontrolle angesehen - erlaubt, die Kolonie zur Suche nach Arbeit zu betreten, vorausgesetzt, sie waren im Besitz von of fiziellen Pässen. ${ }^{23}$

Mit der 1834 in der Kapkolonie erfolgten Abschaffung der Sklaverei führte die Britische Regierung einen Exekutivrat mit vier Beamten und einen Legislativrat ein. Letzterer bestand aus den Beamten, die schon im Exekutiverat saßen und fünf weiteren Bürgern, die vom Gouverneur ernannt wurden und die die Tätigkeit der Beamten unterstützten. ${ }^{24}$ Obwohl dieser Legislativerat in den Gegenständen seiner Kompetenz stark beschränkt

Marais, D., Constitutional Development in South Africa, S. 12.

Muller, C.F.J. (Hrsg.), 500 Jaar Suid-Afrikaanse Geskiedenis, S. 99.

Diese Proclamation ist abgedruckt bei Gordon, R.E./ Talbot, C.J., From Dias to Vorster; Source Material on South African history, 1488-1975, Kapstadt 1983, S. 229; zu ihr: Marais, D., Constitutional Development in South Africa, S. 14.

Muller, C.F.J. (Hrsg.), 500 Jaar Suid-Afrikaanse Geskiedenis, S. 103.

Muller, C.F.J. (Hrsg.), 500 Jaar Suid-Afrikaanse Geskiedenis, S. 115-116.

Vgl. Gordon, R.E./Talbot, C.J., From Dias to Vorster; Source Material on South African history, 1488-1975, S. 229-231. 
war, war den Mitgliedern erlaubt zu diskutieren, und es galt Redefreiheit innerhalb von Zusammenkünften. ${ }^{25}$

Der erste wichtige Schritt - zumindest für die überwiegend weiße Bevölkerung - auf eine demokratischere Form der Regierung hin erfolgte 1853, als die Cape of Good Hope Constitution Ordinance von der Britischen Regierung akzeptiert wurde. ${ }^{26}$ Diese Verfassung wurde am Kap am 1. Juli 1883 in Kraft gesetzt und blieb bis zur 1910 erfolgten Eingliederung der Kapkolonie in die Südafrikanische Union weitestgehend unverändert bestehen. $^{27}$

Mit ihr wurde ein Zweikammer-Parlament mit einem Legislativrat und einem House of Assembly errichtet. Der Legislativerat bestand aus dem Gerichtspräsidenten, der diesem Haus vorstand, und 15 (später 22) gewählten Mitgliedern. Diese wurden auf 10 Jahre gewählt und mußten älter als 30 Jahre alt sein und zusätzlich entweder ein unbewegliches Vermögen im Wert von 2.000 Pfund, oder ein bewegliches Vermögen im Wert von 4.000 Pfund nachweisen. Somit stand damals mehr theoretisch allen ein Wahlrecht, unabhängig von Rasse und Hautfarbe, zu. Das House of Assembly bestand aus 46 Mitgliedern (ab 1887 - 80 Mitglieder), die auf 5 Jahre gewählt worden waren. ${ }^{28}$ Hinsichtlich des aktiven Wahlrechts galt, daß alle männlichen Untertanen der Krone unter der Voraussetzung, daß sie keine kriminelle Vergangenheit aufwiesen und entweder ein Haus von wenigstens 25 Pfund Wert ihr eigen nennen konnten, oder ein jährliches Einkommen von wenigstens 50 Pfund pro Jahr erhielten, wahlberechtigt waren. ${ }^{29}$ Mit diesen Regelungen des aktiven und passiven Wahlrechts sollte die politische Macht in den Händen der "zivilisierten" Wähler gehalten werden, eine Idee, die der Imperialist Cecil John Rhodes fünfzig Jahre später wieder aufgreifen sollte. ${ }^{30}$

Der nächste Meilenstein war 1872 erreicht, als die Regierung durch den Constitution(-al) Ordinance Amendment Act der parlamentarischen Kontrolle unterworfen wurde und der Bestand der Regierung erstmalig vom Vertrauen der Volksvertretung abhing. J.C. Molteno wurde der erste Premierminister dieses Kolonialparlaments, das auf dem Britischen Parlamentssystem basierte.

Kruger, D.W. (Hrsg.), Geskiedenis van Suid-Afrika, S. 179.

Siehe Gordon, R.E.Talbot, C.J., From Dias to Vorster; Source Material on South African history, 1488-1975, S. 239-243.

Muller, C.F.J. (Hrsg.), 500 Jaar Suid-Afrikaanse Geskiedenis. S. 159.

Muller, C.F.J. (Hrsg.), 500 Jaar Suid-Afrikaanse Geskiedenis, S. 158.

Muller, C.F.J. (Hrsg.), 500 Jaar Suid-Afrikaanse Geskiedenis, S. 158.

Gordon, R.E.Talbot, C.J., From Dias to Vorster. Source Material on South African History, 14881975, S. 210. 
In der Kolonie Natal vollzog sich im wesentlichen die gleiche Verfassungsentwicklung. Nach einer kurzen Zeit als Burenrepublik wurde Natal eine Britische Kronkolonie und als solche von 1856 bis 1893 geführt. Im Januar 1893 wurden zwei gewählte Kammern, das House of Assembly und ein Gesetzgebungsrat gebildet, und Natal erhielt insofern weitestgehende Eigenverantwortlichkeit. ${ }^{31}$ Mit den wenigen dort lebenden weißen Siedlern waren die Wahlvoraussetzungen weitaus strenger, um die Anzahl der afrikanischen Wähler zu begrenzen. Der Nachweis von Eigentum oder Vermögen allein genügte nicht, vielmehr waren die Fähigkeit, lesen und schreiben zu können, und das Zeugnis von drei Weißen weitere Voraussetzungen. Dadurch waren in der Wirklichkeit alle Wähler in Natal weißer Hautfarbe. Wegen der indischen Einwanderer, die in die Kolonie strömten und ein Wahlrecht forderten, verabschiedete das Parlament von Natal den Franchise Act of 1896, der all denjenigen ein Wahlrecht versagte, die keine "representative institutions founded on the Parliamentary franchise" hatten, wodurch viele indische Einwanderer ebenfalls vom Wahlrecht ausgeschlossen waren. ${ }^{32}$

In den beiden Burenrepubliken, der Süd-Afrikanischen Republik (Transvaal), gegründet 1852, und dem Oranje Freistaat, gegründet 1854, waren fast demokratische Verfassungen verabschiedet worden. Der Volksraad als Parlament war mit der Einschränkung, daß nur Weiße ein Wahlrecht ausüben konnten und Menschen anderer Hautfarbe von jeglicher politischen Aktivität ausgeschlossen waren, weitestgehend demokratisch gewählt. Erstaunlicherweise wurde dieser auf der Rasse beruhende Ausschluß von der weißen parlamentarischen Demokratie sogar auf alttestamentarische Prinzipien gestützt. ${ }^{33}$

Im Hinblick auf diese zwischen den Burenrepubliken und der Britischen Regierung differierenden Ansichten über die Bedeutung der Rasse zur Gewährung eines Wahlrechts, schlugen mehrere britische Gouverneure und hohe Beamte die Gründung einer Föderation südafrikanischer Staaten vor, so vor allem die Bemühungen von Grey, Carnarvon, Rhodes and Milner. Das Vorhaben, dieses Ziel friedlich zu erreichen, scheiterte jedoch, und einzig durch die militärische Niederwerfung der Burenrepubliken im Jahre 1902 entstand eine engere Union dieser Staaten. ${ }^{34}$ Kronos, 1, Bellville 1979, S. 30.

34 Vgl. Barnard, L., "The attempts of the British Government to establish a federation in Southern Africa during the second half of the nineteenth century", in: Kriek, D.J. (Hrsg.), Federalism: The Solution? Principles and Proposals, Pretoria 1992, S. 117-133 und Gordon, R.E./Talbot, C.J., From Dias to Vorster: Source Material on South African history, 1488-1975, S. 253-257, 259-264. 
Die Vorteile einer engeren politischen Verbindung zwischen den vier Britischen Kolonien wurden eingehend in der Konferenz erörtert, die 1908 in Kapstadt stattfand. ${ }^{35}$ Diese Konferenz, auf der die verschiedensten politischen Parteien durch die wirtschaftlichen Zustände zusammenkamen, führte zur Einberufung einer Nationalversammlung, die eine Verfassung erarbeiten sollte. Dazu gab es von Oktober 1908 bis Mai 1909 verschiedene Treffen. Dabei wurde auch der Aspekt des Föderalismus beraten; die Mehrheit jedoch entschied sich für eine politische Union mit einer starken Zentralregierung. Das Parlamentssystem war, entsprechend dem Westminster-System, mit einem Ober- und Unterhaus unter absoluter Geltung parlamentarischer Souveränität ausgestaltet, so daß die Legislative frei von richterlicher Kontrolle war.

Um die Unterschiede im Wahlrecht zwischen Kap und Natal auf der einen Seite und Transvaal sowie dem Oranje-Freistaat auf der anderen Seite in Einklang zu bringen, erhielten die neuen Provinzen der Südafrikanischen Union einige föderale Rechte. So konnte im Transvaal kein Nicht-Weißer wählen; in der Kap-Provinz jedoch wurden die Coloured und Afrikaner nicht vom aktiven Wahlrecht ausgeschlossen. Im Gegensatz dazu stand nur Weißen allein ein passives Wahlrecht zu. Diese Prinzipien und der gleiche Status der beiden Amtssprachen Englisch und Holländisch konnten nur mittels einer Zweidrittelmehrheit einer Sitzung beider Häuser geändert werden. Sowohl die teilweise Nichtgewährung des aktiven Wahlrechts, als auch der Ausschluß der farbigen und schwarzen Menschen von den höchsten Staatsämtern führte zu Protesten. Daher entsandten die Vereinigungen der Coloured "African Political Organisation" und der "Black South African Native Convention" Delegationen, die nach Großbritannien reisten, um dort gegen die neue Verfassung ihren Protest zu äußern. Dies war aber vergeblich. Der South Africa $\mathrm{Act}^{36}$ wurde am 20. September 1909 vom Britischen Parlament verabschiedet und trat am 31. Mai 1910 in Kraft. ${ }^{37}$ Diese Verfassung blieb im wesentlichen bis 1956 unverändert.

Nachdem die Nationale Partei die Politik der Apartheid 1948 eingeführt hatte, wurden die farbigen Wähler 1956 in der Kap-Provinz aus dem Wahlverzeichnis gelöscht. Dies gelang nur durch eine Vergrößerung des Senates, da nur so eine 2/3 Mehrheit erreicht werden

Van Jaarsveld, F.A., 100 basiese dokumente by die studie van die Suid-Afrikaanse geskiedenis, 1648-1961, Kapstadt 1971, S. 181-182.

Der gesamte Inhalt ist (in Englisch) abgedruckt bei Van Jaarsveld, F.A., 100 basiese dokumente by die studie van die die Suid-Afrikaanse geskiedenis, 1648-1961, S. 182-201.

37

Der Ausschluß einer Rasse oder ethnischen Gruppe ist in neuzeitlichen Verfassungen nicht nur auf Südafrika begrenzt. Erst 1985 schlossen Artikel 27 und 22 der Liberianischen Verfassung Weiße vom Erwerb der Staatsbürgerschaft aus. Siehe dazu: Heese, H.F., "Ras en Etnisiteit; Suid-Afrikaanse perspektiewe in 1938 en 1988", Kronos, 16, 1989, S. 42-51. 
konnte. ${ }^{38}$ Als dann 1961 die bisher monarchische Verfassung in der republikanischen Verfassung aufging, änderte sich bis auf die Ersetzung des Monarchen durch einen Staatspräsidenten nichts wesentliches. ${ }^{39}$

1983 wurde dann die Verfassung ergänzt, um die asiatischen und farbigen Menschen in einem Dreikammernparlament und einem Präsidialrat, der den alten Senat ersetzte, zu integrieren. ${ }^{40}$ In der Verfassungswirklichkeit blieb der Einfuß der Mischlinge und Asiaten aber gering, was zum einen daran lag, daß sie gegenüber den Weißen in geringerer Zahl in Parlament und Präsidialrat vertreten waren und so eigentlich nichts wirklich mitentscheiden konnten, und zum anderen die Finanzausstattung zur Gestaltung ihrer eigenen "Gruppenangelegenheiten" vom Wohlwollen der weißen Parlamentsmehrheit abhing. Diese politische Initiative genügte jedoch insbesondere nicht der immer stärker erhobenen Forderung nach einer nicht rassistischen Verfassung, die, auf einem allgemeinen Wahlrecht basierend, allen Menschen - und damit eben gerade auch der schwarzen Bevölkerungsmehrheit - die gleichen Rechte zugestehen müsse.

\section{Die Entstehung der Neuen Interimsverfassung}

Nachdem Südafrika immer stärker unter den internationalen Sanktionen und dem weltweiten wirtschaftlichen Abschwung zu leiden hatte, im Inneren die politisch motivierten Gewalttaten zunahmen und in der Nationalen Partei ein Führungswechsel stattgefunden hatte, setzte sich in den politischen und wirtschaftlichen Führungszirkeln Südafrikas immer mehr die Erkenntnis durch, daß die Politik der Apartheid ein Ende haben müsse. Dieser von oben einsetzende Umdenkungsprozeß wurde vom erst kurz im Amt befindlichen Staatspräsidenten De Klerk mit seiner Parlamentsrede vom 2. Februar 1990 in die breite Öffentlichkeit getragen. In ihr kündigte De Klerk an, das Verbot diverser Parteien und Organisationen aufzuheben ${ }^{41}$ und die politischen Gefangenen freizulassen ${ }^{42}$, um die Überwindung der Apartheid einzuleiten und so Südafrika ein neues Angesicht zu geben. Danach begann ein mehrjähriger Verhandlungsprozeß, in dem neben der Frage des

Das Vorspiel dieses Ereignisses wird diskutiert durch: Scher, D.M., "The court of errors; a study of the High Court of Parliament crisis of 1952", Kronos, 13, 1988, S. 23-40.

39 Marais, D., Constitutional Development in South Africa, S. 33-35.

40 Aus der umfangreichen Literatur zu der 1983 Verfassung siehe nur Basson, D/Viljoen, H. South African Constitutional Law, Cape Town 1988; den Bericht der International Commission of Jurists, South Africa: Human Rights and the Rule of Law, London 1988 und von der Ropp, Klaus, Die neue Verfassung der Republik Südafrika - von Westminster nach Southminster, in VRÜ 1984, S. 194ff.

41 Bis dahin waren der ANC, Pan Africanist Congress, South African Communist Pary, Umkhonto weSizwe (die paramilitärischen Einrichtungen des ANC) und Azanian People's Liberation Army (die der PAC) verboten gewesen.

42 Nelson Mandela verließ 9 Tage später am 11. Februar sein Gefängnis, nach insgesamt 27 Jahren Haft. 
Machttransfers auch die Gestalt eines dann veränderten Südafrikas erörtert werden sollte. Die insofern geführten Verhandlungen fanden zunächst zwischen 19 politischen Parteien und Gruppierungen statt. Dabei kam den teilnehmenden Parteien zwar ein gleiches Stimmgewicht zu, die Hauptakteure waren jedoch die damals regierende Nationale Partei, der Afrikanische Nationalkongreß, die Inkatha Freiheitspartei, die Demokratische Partei und die Freiheitsfront, um die sich die anderen Verhandlungsteilnehmer gruppierten. Diese Verhandlungen begannen offiziell am 20. Dezember 1991 unter der Bezeichnung CODESA (Convention for a Democratic South Africa) ${ }^{43}$ und wurden in fünf Arbeitsgruppen aufgenommen, die dann ihre Ergebnisse der Plenarsitzung von CODESA 2 am 15. und 16. Mai 1992 vorlegten. Zwischenzeitlich erhielt Staatspräsident De Klerk diesen eingeschlagenen Weg der Reformen, an dessen Ende der Verlust der weißen Vorherrschaft stehen würde, in einem am 17.3.1992 abgehaltenen Referendum der Weißen mit $68,7 \%$ bestätigt. Da aber auf der Plenarsitzung keine Einigkeit über die Frage erzielt werden konnte, welche Stimmenanzahl für die Annahme der Beschlüsse einer Verfassungsgebenden Versammlung nötig sei, wurde CODESA 2 vertagt.

Zwar scheiterte CODESA dann gänzlich nach einem Blutbad in Boitpatong, für das der ANC die Polizei und die Regierung verantwortlich machte und daraufhin die Verhandlungen am 23. Juni 1992 abbrach. In der Folgezeit setzte jedoch erneut eine Annäherung zwischen der Regierung und dem ANC ein, die einen neuen Abschnitt der Verhandlungen einleiten sollte und zu einer gemeinsamen Erklärung zwischen der Regierung und dem ANC führte, die als "Record of Understanding" bekannt geworden ist. Den Schlußpunkt bildeten dann die von 26 Parteien am 1. April 1993 aufgenommenen Mehrparteienverhandlungen, der sog. Multi-Party Negotiating Process, wobei die schwierige Verhandlungsarbeit der detaillierten Verfassungserarbeitung in 7 technischen Ausschüssen geleistet wurde, die sich ganz überwiegend aus hochkarätigen Mitgliedern zusammensetzten. ${ }^{44}$

Eine der größten Differenzen zwischen den Verhandlungsteilnehmern betraf die Grundsatzfrage, welchen Regelungsauftrag und damit welchen Inhalt die neue Verfassung erhalten sollte. Dazu standen sich zwei gegensätzliche Auffassungen gegenüber. Die eine Ansicht, vom ANC vertreten, ging dahin, daß die Mehrparteienverhandlungen aufgrund ihrer unzureichenden Repräsentanz allerhöchstens eine reine Übergangsverfassung aushandeln könnten und einer gewählten Volksvertretung die endgültige Ausarbeitung der neuen Verfassung zukomme. Die andere Ansicht, vor allem die Inkatha Freiheitspartei und die Regierungsvertreter von Bobhutatswana und der Ciskei vertreten, bestand darauf, daß die Verfassung das Ergebnis der Mehrparteienverhandlungen darstellen sollte und so

Zur Struktur und zur inhaltlichen Ausgestaltung der Arbeit von CODESA vgl: Klug, Heinz, A report on the Convention for a Democratic South Africa, in: SAJHR 1992 (Vol. 8), Part 2, S. 249-269.

44

Insbesondere arbeiteten in ihnen auf allen Verhandlungsseiten zahlreiche Professoren der Juristischen Fakultäten mit. 
komplett wie irgend möglich gestaltet werden muß. Zwischen diesen beiden Positionen bewegte sich die Regierung. Der schlußendlich erarbeitete Kompromiß besteht nunmehr darin, daß die Verfassung nur für fünf Jahre Gültigkeit erlangen soll und in dieser Zeit eine gewählte Verfassungsgebende Versammlung eine endgültige Verfassung unter Beachtung vereinbarter Verfassungsprinzipien zu erstellen hat. Diese fast zwei Jahre dauernden Verhandlungen fanden mit den Beschlüssen von Kempton Park über den Text der neuen Verfassung am 18. November 1993 ihren Abschluß.

Diese Verfassung wurde dann vom alten Drei-Kammern-Parlament auf einer Sondersitzung im Dezember 1993 verabschiedet und von Staatspräsident De Klerk am 28. Januar 1994 in der Government Gazette verkündet. Doch selbst danach wurden noch Ergänzungen der Verfassung vorgenommen, so im 1. Amendment, das die Kompetenzen der Provinzen stärkte und, den Bemühungen der Freiheitsfront entgegenkommend, einen Volksstaat Council errichtete, der der Verfassungsgebenden Versammlung Vorschläge zur Errichtung eines Volksstaats ${ }^{45}$ unterbreiten kann. Die letzte Änderung der Verfassung wurde noch zwei Tage vor Inkrafttreten der Verfassung durch das alte Parlament vorgenommen, um - worauf oben schon hingewiesen wurde - die verfassungsrechtliche Absicherung der Institution, der Rolle und Autorität, die der Zulu-Monarchie zukommt, zu gewährleisten und so der Inkatha Freiheitspartei die Teilnahme an den Wahlen unter Verzicht auf Gewalt zu ermöglichen.

\section{Der Verfassungsinhalt}

Die aus insgesamt 251 Artikeln bestehende Verfassung ist, unter Voranstellung einer Präambel und unter Hintenanfügung von 7 Anhängen (Schedules), in 16 Kapitel eingeteilt. Im Hinblick darauf, daß die Neue Verfassung auf einem Kompromiß zwischen den Parteien, die sich an den Mehrparteienverhandlungen beteiligten, beruht und versucht die Unterschiede der verschiedenen, teilweise gegensätzlichen Ansichten aufzulösen und in Einklang zu bringen, ist die Verfassung außerordentlich lang und komplex gestaltet und liest sich manchmal wie ein detaillierter Vertrag, der für alle Eventualitäten Strukturen odẹr Verfahren festlegt. gegründet werden soll, um sich selbstverwaltend eine eigenständige Identität zu bewahren - diese Idee wurde auch als Gründung "weißer Homelands" bezeichnet. 
Die Neue Übergangsverfassung darf als eines der wichtigsten Übergangsinstrumente ${ }^{46}$ angesehen werden und ist - überblicksartig dargestellt - außergewöhnlich, indem sie, die wesentlichen Strukturen dieser Übergangszeit regelnd, bestimmt,

- daß die bisherigen Provinzen, die sich selbstverwaltenden Homelands und die nominell unabhängigen TBVC-Staaten ${ }^{47}$ aufgehört haben zu existieren und das Land in 9 neue Provinzen unterteilt worden ist;

- daß die Regierungsgewalt in den nächsten 5 Jahren durch ein Government of National Unity ausgeübt wird, an dem alle mit mindestens $5 \%$ in der National Assembly vertretenen Parteien beteiligt sind und das nach dem Konsensprinzip ausgeübt werden soll;

- daß dem Parlament zusätzlich zu seiner gesetzgebenden Gewalt zugleich auch als Verfassungsgebenden Versammlung die Aufgabe zukommt, binnen 2 Jahren eine dann endgültige Verfassung mit Zweidrittel-Mehrheit zu verabschieden, die sich in Übereinstimmung mit den in Schedule 4 enthaltenen Verfassungsprinzipien befinden muß, was das neu geschaffene Verfassungsgericht überprüft;

- daß Parlament, Regierung und Verwaltung durch die in Abschnitt 3 der Interimsverfassung enthaltenen Grundrechte erstmalig in der Ausübung ihrer Gewalt begrenzt sind und jedes staatliche Handeln vor Gerichten auf dessen Vereinbarkeit mit diesen Grundrechten überprüft werden kann;

- daß eine Vielzahl von Kommissionen errichten werden, um die Geltung der Menschenrechte, des Rechts auf Gleichheit zu fördern und Polizei, Armee, den Öffentlichen Dienst und die Verwaltung zu reformieren und effizienter zu gestalten.

\section{Der Geist der Verfassung}

Hinsichtlich des grundlegenden Selbstverständnisses spiegelt sich bereits in der Präambel der Geist des Gesamtwerkes deutlich wider. Im Gegensatz zur Präambel der Verfassung von 1983, in der die Theorie des Separate Development, die das ideologische Fundament der Politik der Apartheid bildete, als Staatsziel anerkannt wurde ${ }^{48}$, wird die Notwendigkeit der Schaffung einer neuen Ordnung betont, die durch eine allgemeine Staatsbürgerschaft, die Gleichheit aller Menschen, unabhängig von Geschlecht und Rasse, und die Ausübung der Grundrechte geprägt sein soll. Das Schlußwort der Verfassung benennt den konkreten Weg dieses Übergangs aus der konfliktträchtigen, ungerechten und Leid schaffenden Vergangenheit in eine gemeinsame Zukunft dahingehend, daß durch Wiedergut-

Andere Übergangsinstrumente waren die Gesetze, die zur Ermöglichung und Einhaltung freier und fairer erster allgemeiner Wahlen verabschiedet wurden, so der Transitional Executive Council Act, der Independent Media Commission Act, Independent Broadcasting Authority Act, Independent Electoral Commission Act und der Electoral Act.

8 Republic of South Africa Constitution Act 110 of 1983 mit der Formulierung: "To respect, to further and to protect the self determination of population groups and nations". 
machung und durch eine Erneuerung der Gesellschaft die nationale Einheit, das Wohlergehen der Menschen und Friede in Südafrika verwirklicht werden sollen.

Als erste Verwirklichungsstufe und unabdingbare Voraussetzung dieses Prozesses sind die Bestimmungen der ersten beiden Kapitel den anderen Verfassungsregelungen vorangestellt worden. In Art. 1 wird zunächst das Staatsterritorium, das die im einzelnen in Anhang 1 Teil 1 aufgeführten Gebiete umfaßt, bestimmt. Wie sich aus den dort aufgeführten Gebieten ergibt, sind die sich selbstverwaltenden Homelands und die sog. "unabhängigen" TBVC-Staaten aufgelöst und in einen einheitlichen Staat wiedereingegliedert worden ${ }^{49}$, so daß damit der ehemaligen Politik der Separate Development jegliche Legitimation entzogen worden ist und eine gewisse Wiedergutmachung der erfolgten Ausgliederung bereits stattgefunden hat.

In Art. 2 sind die emotional bedeutsamen Fragen der südafrikanischen Flagge und Hymne im Sinne einer das Alte mit dem Neuen verbindenden Weise geregelt worden. ${ }^{50}$ Die wichtige Frage der Anerkennung der Vielfältigkeit Südafrikas findet sich dann in Art. 4, der neben den bislang geltenden Amtssprachen Afrikaans und Englisch neun weitere "schwarze" Sprachen (isiNdebele, Sesotho sa Leboa, Sesotho, siSwati, Xitsonga, Setswana, Tshivenda, isiXhosa und isiZulu) zu Amtssprachen auf nationaler Ebene erhebt und die Förderung aller offiziellen Sprachen und der Mehrsprachlichkeit einem Pan South African Language Board anvertraut, das darüber hinaus auch die von Minderheiten gesprochenen Sprachen wie Hindi, Portugiesisch und sogar Deutsch fördern soll.

Indem darüber hinaus, mit aus südafrikanischer Sicht revolutionären Worten, ${ }^{51}$ in Art. 4 alle Staatsgewalt der Bindung an die Verfassung unterworfen wird und die Verfassung den höchsten Rechtsstatus erhält, bricht Südafrika mit der seit 1910 herrschenden, britisch geprägten Verfassungstradition, die der uneingeschränkten Souveränität des

Dies geschieht , indem die Gesetze, die den ehemaligen Homelands deren Unabhängigkeit gewährten, einfach wieder aufgehoben worden sind; siehe dazu im Schedule 7: Act No. 100 of 1976, Act No. 89 of 1977, Act No. 107 of 1979 und Act No. 110 of 1981 und zur Frage, wie eine Wiedereingliederung, über die im Ergebnis keine Zweifel bestanden, die Diskussion im Schrifttum: Wichers, Marinus, Reincorporation of the TBVC countries - international law practice and constitutional implications in: SAYIL 1990/91 S. 119-126 und Carpender, Gretchen, Reincorporation of Transkei into the Republic of South Africa, the constitutional implications in: SA Public Law 1990, S. 250-255.

Die Auswahl der Staatsflagge und der Nationalhymne obliegt dem Staatspräsidenten; auf grund einer Empfehlung des Transitional Executive Council (TEC) sind durch den ehemaligen Staatspräsidenten De Klerk eine neue Flagge und als Hymnen "Die Stem" und "Nkosi Sikelel'iAfrica" ausgewählt worden.

Vgl. dazu nur: Victor Southwell und Jan H van Rooyen, The procedural management of constitutional issues in criminal trials in a future South Africa: a cost-effectiveness study, in: CILSA 1993 (Vol. 26), Nr.3, S. 303 - 351 und CRM Dlamini, The South African Law Commission's Working Paper on Group and Human Rights: towards a bill of rights for South Africa?, in: SA Publiekreg/Public Law 1990 (Vol.107), S. 91-100. 
Parlamentes den Vorrang vor einer Bindungswirkung der Verfassung einräumte und niemals anerkannte, daß die Verfassung als grundlegendes Recht die höchste Autorität zwischen dem Staat und den seiner Gewalt unterworfenen Bürgern darstellt.

Schließlich wurde der Regelung der Staatsbürgerschaft und des Wahlrechts ein eigenes Kapitel eingeräumt, was die Bedeutung dieser beiden Gegenstände im Hinblick auf die Praxis in der Vergangenheit nur noch unterstreicht. Indem nämlich eine allgemeine Staatsbürgerschaft wiederhergestellt und allen mindestens 18 Jahre alten südaf rikanischen Staatsbürgern ein Wahlrecht zuerkannt wird, erhält die Anti-Apartheidsbewegung in ihrem über vier Jahrzehnte währenden Kampf eine formelle Bestätigung und Anerkennung. Entgegen dieser verfassungsrechtlichen Einräumung eines allgemeinen Wahlrechts waren aber aufgrund der Regelung in $\S 16$ des Electoral Acts Strafgefangene, die wegen schwerer Gewaltdelikte Strafen verbüßten, von der Ausübung ihres Wahlrechts in den Wahlen vom April 1994 ausgeschlossen, was insbesondere der ANC im Vorfeld der Wahlen anprangerte und von der Regierung mit der dieser Straftat folgenden Verwirkung dieses Rechts gerechtfertigt wurde.

\section{Das Parlament}

Die Gesetzgebungskompetenz steht nach Art. 36 dem Parlament zu, das aus zwei Häusern, der Nationalversammlung und dem Senat, besteht. Die Nationalversammlung besteht nach Art. 40 Abs. 1 aus 400 Abgeordneten, die nach den Grundsätzen einer in Schedule 2 und dem Electoral Act $^{52}$ näher bestimmten Verhältniswahl gewählt werden, ${ }^{53}$ so daß damit auch das seit Bestehen der Südafrikanischen Union (1910) praktizierte absolute Mehrheitswahlsystem abgelöst worden ist. Nach § 2 Schedule 2 werden von den 400 Sitzen in der Nationalversammlung 200 Sitze nach Provinzlisten und 200 nach Nationallisten besetzt, um eventuell auftretende Disproportionalitäten zu korrigieren. Hervorzuheben ist, daß auf eine explizit formulierte Minderheiten-Sperrklausel zugunsten der Integration der kleineren Parteien verzichtet wurde, da gerade die kleinen Parteien, die verschiedensten Minderheiten repräsentierend, in den Übergangsprozeß integriert werden sollten, um so auch mögliche Konflikt- und Gewaltpotentiale einzudämmen. Die in jedem Verhältniswahlsystem immanent vorhandene Sperrklausel, die sich aus der Stimmenanzahl ergibt, die für einen Abgeordnetensitz benötigt wird, ist sehr niedrig — insgesamt wurden 19.340.416 gültige Stimmen abgegeben, so daß die Quote für einen Sitz in der Nationalversammlung 48.231 Stimmen beträgt, was ungefähr einem Stimmenanteil von

52

53 
0,25\% entspricht. Diese den kleinen Parteien günstige Regelung befähigte 4 Parteien dazu, in die Nationalversammlung einzuziehen. ${ }^{54}$

Die Amtszeit des durch die ersten allgemeinen Wahlen am 26.-29. April 1994 gewählten Parlaments ist nach Art. 38 auf die Übergangszeit von 5 Jahren beschränkt, was sich neben der Formulierung in Art 38 Abs. 1 auch aus Abs. 4 ergibt, der bei einer Neuwahl die Amtszeit des Parlaments auf die noch nicht abgelaufene Fünfjahreszeit beschränkt.

Während dieser Übergangszeit kann eine Auflösung des Parlaments mit anschließenden Neuwahlen nur durch den Staatspräsidenten unter den Voraussetzungen erfolgen, daß es dem Parlament entweder nicht gelingt, eine neue Verfassung binnen zwei Jahren anzunehmen ${ }^{55}$, oder daß das Parlament dem Kabinett einschließlich des Präsidenten das Mißtrauen ausspricht ${ }^{56}$ oder nur-dem Kabinett das Mißtrauen ausspricht, und sich der Staatspräsident für diesen Fall für eine Parlamentsauflösung entscheidet.

Die Stellung der Abgeordneten und Senatoren wird zum einen dadurch geprägt, daß ein Doppelmandat zwischen beiden Häusern des Parlaments und zwischen diesen und dem Provinzparlament ausgeschlossen ist ${ }^{57}$, den einzelnen Parlamentarier keine strafrechtliche Verantwortung und keine zivilrechtliche Haftung für das trifft, was er "in or before or to Parliament or any comittee thereof" gesagt, getan und unterstützt hat oder daraus entstanden ist. ${ }^{58}$ Darüber hinaus werden die Parlamentarier aus der Staatskasse bezahlt ${ }^{59}$; auch steht ihnen ein Pensionsanspruch zu.

Auf der anderen Seite enthält die Verfassung die für uns eigentümliche Regelung, daß ein Abgeordneter dann sein Mandat verliert, wenn er aufhört, Mitglied der ihn nominierenden Partei zu sein, was den Parteien ein erhebliches Disziplinierungsinstrument in die Hand legt, da bei einem der Partei mißliebigen Verhalten neben dem Parteiausschluß immer auch der Mandatsverlust droht. Diese Regelung, eine direkte Abhängigkeit von Abgeordnetenmandat und Parteimitgliedschaft, zeigt die Entscheidung zugunsten einer Parteiendemokratie und ist nur insofern erklärbar, als damit verhindert werden soll, daß einzelne Abgeordnete die Lager zur Erzielung persönlicher Vorteile kreuzen, was im alten Drei-

Für die National Assembly: Freedom Front 2,17\% = 9 Sitze; Democratic Party 1,73\% = 7 Sitze; Pan Africanist Congress of Azania 1,25\% = 5 Sitze; African Christian Democratic Party 0,45\% = 2 Sitze Art. 43 Zi. e; 51 Abs. 1 Zi. e; 133 Abs. 1 Zi. e; 179 Abs. 5 Zi.b

Art. 55 Abs. 3; über diese Indemnität hinaus bleibt es einem einfachen Gesetz vorbehalten, Regelungen zur Imunität der Abgeordneten und Senatoren zu treffen. Nach einem Empfehlung des TEC betragen die Abgeordnetenbezüge ZAR 193.000 pro Jahr. 
Kammern-Parlament häufiger vorgekommen war, um so einem sich nahe der ZweidrittelMehrheit befindlichen ANC eine verfassungsändernde Mehrheit zu beschaffen.

Eine ausschließliche Gesetzgebungskompetenz steht dem Parlament nach Art. 37 für alle Materien zu, die nicht explizit den Provinzen in Art. 126 Abs. 1 i.V.m. Schedule 6 als Gesetzgebungskompetenzen eingeräumt sind. Hinsichtlich dieser vorrangig den Provinzen eingeräumten Gesetzgebungskompetenz steht dem Parlament aber unter den weit gefaßten Voraussetzungen des Art. 126 Abs. 3 eine konkurrierende Gesetzgebungskompetenz zu. Wie bereits verdeutlicht wurde, ist das Parlament durch die Verfassung als höchstes Recht und insbesondere die in ihr enthaltenen Grundrechte in der Ausübung seiner Souveränität gebunden.

Der Senat bildet sich aus jeweils 10 Senatoren für jede der 9 Provinzen und umfaßt somit 90 Senatoren. Diese werden jedoch nicht durch das jeweilige Provinzparlament gewählt, wie es noch der 10. Report des Technischen Komitees für Verfassungsfragen vorschlug, sondern entsprechend Art. 48 Abs. 2 der Verfassung von den im jeweiligen Landesparlament vertretenen Parteien in der Anzahl ernannt, die dem Verhältnis ihrer im Landesparlament errungenen Abgeordnetensitze entspricht. ${ }^{60}$ Die so zu ernennenden Senatoren brauchen nicht gewählte Abgeordnete eines Provinzparlaments zu sein, müssen aber der sie ernennenden Partei angehören. ${ }^{61}$

Was das Gesetzgebungsverfahren angeht, so unterscheidet die Verfassung einfache Gesetze, Finanzgesetze, Gesetze, die Provinzangelegenheiten betreffen und verfassungsändernde Gesetze. Hinsichtlich der einfachen Gesetze bestimmt Art. 59, daß diese sowohl in der Nationalversammlung als auch im Senat eingebracht werden können und der Annahme in beiden Häusern bedürfen. Bei der Annahme in nur einem der Häuser und der Zurückweisung durch das andere, soll das Gesetz in einem gemeinsamen Ausschuß beider Häuser beraten und ggf. ergänzt werden, um danach in einer gemeinsamen Sitzung beider Häuser mit einfacher Mehrheit der gesamten Anzahl der Mitglieder beider Häuser verabschiedet zu werden.

Am Beispiel der Western Cape Province dargestellte Berechnung nach der in Art. 48 Abs. 2 Buchstab. a und $b$ enthaltenen Formel:

Sitzverteilung im Landesparlament: National Party:

Af rican National Congress: $\quad 14$

Democratic Party:

Freedom Front:

African Christian Dem. Party:

Gesamt:

\begin{tabular}{|c|c|c|c|c|}
\hline \multirow[b]{2}{*}{ Formel } & \multicolumn{4}{|c|}{ zu nominierende Senatoren } \\
\hline & ganze & & Rest & Anzahl \\
\hline $23 \times 10: 43$ & $=\underline{5}$ & und & 0,3488 & 6 \\
\hline $14 \times 10: 43$ & $=$ & und & $\overline{0,2558}$ & 3 \\
\hline $3 \times 10: 43$ & $=$ & und & 0,6976 & $\mathbf{1}$ \\
\hline $1 \times 10: 43$ & $=$ & und & $\overline{0,2325}$ & 0 \\
\hline $1 \times 10: 43$ & $=$ & und & 0,2325 & 0 \\
\hline & & & Gesamt: & 10 \\
\hline
\end{tabular}

Umkehrschluß aus Art. 51 Abs. 1 Zi. b, der bestimmt, daß ein Senator seinen Sitz verliert, wenn er aufhört, der ihn nominierenden Partei anzugehören. 
Finanzgesetze dagegen können nach Art. 60 nur in die Nationalversammlung eingebracht werden und werden nach Beratung in einem gemeinsamen Ausschuß beider Häuser und nach Anhörung der Empfehlungen der Financial and Fiscal Commission durch die Nationalversammlung angenommen, wobei eine eventuelle Zurückweisung, beantragte Änderungen oder eine Nichtbehandlung innerhalb von 30 Tagen durch den Senat dazu führt, daß das Gesetz in die Nationalversammlung zurückverwiesen wird, die dieses Gesetz dann endgültig verabschiedet.

Gesetze, die die Grenzen oder die Ausübung oder Erfüllung der Machtbefugnisse und Funktionen der Provinzen betreffen, benötigen nach Art. 61 die mehrheitliche Zustimmung des Senats und für den Fall, daß eine einzelne Provinz von dem Gesetz betroffen ist, die mehrheitliche Zustimmung der diese Provinz vertretenden Senatoren.

Mögliche Verfassungsänderungen bedürfen nach Art. 62 der qualifizierten ZweidrittelMehrheit einer grundsätzlich gemeinsamen Sitzung von Nationalversammlung und Senat. Betrifft die Verfassungsänderung jedoch die in Art. 126 und Art. 144 verankerten Kompetenzen der Provinzen, so ist dazu eine Zweidrittel-Mehrheit in getrennten Sitzungen beider Häuser erforderlich.

\section{Der Präsident und das Kabinett}

Das 6. Kapitel etabliert für die Übergangszeit bis 1999 die besondere Regierungsform des Government of National Unity, das sich dadurch auszeichnet, daß es den Parteien, die eine relevante Minderheit in der Nationalversammlung bilden, einen verfassungsrechtlichen Anspruch auf Teilhabe an der Regierung durch die Entsendung von Executive Deputy Präsidenten und Ministern einräumt und so von Verfassungs wegen eine große Koalition nahezu aller Parteien zur Erneuerung Südafrikas bildet.

Das Kapitel beginnt mit dem Staatspräsidenten, dem in der Übergangsverfassung eine sehr bedeutsame Stellung zukommt. Der von der Nationalversammlung auf fünf Jahre gewählte Staatspräsident $^{62}$ ist repräsentatives Staatsoberhaupt, Regierungschef und Oberbefehlshaber der Streitkräfte. Dies führt dazu, daß er nach Art. 81 als Regierungschef für die Einhaltung der Bestimmungen der Verfassung durch die Exekutive verantwortlich ist und als Staatsoberhaupt die Verfassung als das ranghöchste Recht verteidigen und hüten soll. Art. 82 beschreibt im Einzelnen die umfangreichen Aufgaben, Ermächtigungen

Im Gegensatz zu der Verfassung von 1962 und in Übereinstimmung mit der Verfassung von 1982 hängt die Amtszeit direkt mit dem Bestand des Parlaments zusammen. Nach einer Auflösung des Parlaments verbleibt er nach Art. 80 Abs. 1 b nur solange im Amt, bis ein neuer Staatspräsident amtiert. 
und Pflichten des Staatspräsidenten, die von Unterzeichnung und Ausfertigung von Gesetzen, der Akkreditierung von ausländischen Diplomaten und dem Recht, Begnadigungen auszusprechen, bis hin zur Ernennung der Minister, der Einsetzung von besonderen Kommissionen und zur Auflösung des Parlaments reichen.

Hinsichtlich all seiner im Zusammenhang mit seiner Stellung als Regierungschef stehenden Aufgaben soll er Entscheidungen in Konsultationen mit seinen beiden Executive Deputy Präsidenten und dem Kabinett treffen. "In Konsultationen" bedeutet, daß entsprechend dem Geist, der dem Konzept des Government of National Unity zugrunde liegt, zu jeder Zeit versucht wird, Übereinstimmung zu erzielen (Art. 88 Abs. 4 und 5), wobei im Fall, daß eine solche nicht erreichbar ist, der Staatspräsident die letzte Entscheidungsgewalt besitzt. Die bereits erwähnten Executive Deputy Präsidenten können nach Art. 84 von den Parteien gestellt werden, die wenigstens 80 Sitze in der Nationalversammlung innehaben.

Das Kabinett besteht aus dem Staatspräsidenten, den beiden Executive Deputy Präsidenten und nicht mehr als 27 Ministern ${ }^{63}$. Jede Partei, die wenigstens 20 Sitze - entsprechend 5\% - der Sitze in der Nationalversammlung inne hat, ist nach Art. 88, entsprechend dem Verhältnis ihrer Sitzanzahl in der Nationalversammlung, berechtigt, Minister im Kabinett zu stellen. Die Verteilung der Ressorts auf die einzelnen Minister erfolgt dann durch den Staatspräsidenten nach Beratung mit seinen Stellvertretern.

\section{Die Grundrechte}

Kapitel 3 enthält 25 Grundrechte, die während der Verhandlungen für die Phase des Übergangs als wesentlich und verfassungsrechtlich schützenswert angesehen wurden. $\mathrm{Zu}$ dieser, während der Mehrparteienverhandlungen getroffenen Entscheidung, welche Rechte Aufnahme in den Grundrechtskatalog finden sollten, standen sich eine minimalistische und eine optimalistische Position gegenüber. Die Minimalisten argumentierten, daß die Liste nur die Rechte umfassen sollte, die für den politischen Prozeß des Übergangs unerläßlich seien. Die Optimalisten hingegen traten für die vollstmögliche Aufnahme von Rechten ein. Insof ern wurde deutlich, daß der Schutz der Menschenrechte eine Seite des Kampfes um politische Macht war, der insbesondere zwischen der regierenden National Party auf der optimalistischen Seite und dem ANC auf der minimalistischen Seite geführt wurde. Der schlußendlich erzielte Verhandlungskompromiß führte zu einem unvollständigen Grundrechtskatalog, wobei die diesem Kapitel zugrundeliegende Philoso-

In der gegenwärtigen Regierung wurden 27 Minister ernannt, davon gehören 18 dem ANC, 6 der Nationalen Partei und 3 der Inkatha-Freiheitspartei an. 
phie in den Art. 7 (Application) und Art. 35 (Interpretation) deutlich zum Ausdruck kommt.

In enger Anlehnung an die Canadian Charter of Rights and Freedoms ${ }^{64}$ sind die Formulierungen der einzelnen Grundrechtsartikel möglichst verständlich und vom Schutzbereich umfassend gehalten und entsprechen den internationalen Anforderungen. Die einzelnen Grundrechte reichen vom an erster Stelle genannten Recht auf Gleichheit und Freiheit von Diskriminierung (Art. 8), über den Schutz des Lebens (Art. 9) bis hin zu dem Recht auf eine Grundschulausbildung und auf gleichen Zugang zu Bildungsinstitutionen (Art. 32).

Die besonders wichtige Frage der Einschränkbarkeit der Grundrechte wird für alle einzeln verbürgten Rechte in Art. 33 geregelt. Danach ist die Beschränkbarkeit der Grundrechte im Rahmen der Verhältnismäßigkeit auch dadurch begrenzt, daß die einschränkenden Gesetze allgemein sein müssen und den Wesensgehalt des betroffenen Grundrechts unangetastet lassen.

Auf welche Weise Gerichte Schutz vor Eingriffen der Legislative und Exekutive gewähren, bestimmt sich aus Art. 7 Abs. 4 i.V.m. Art. 98 und Art. 99. Dabei eröffnet Art. 7 Abs. 4 nicht nur den in ihren Grundrechten selbst Beeinträchtigten die Klagemöglichkeit, sondern gestattet auch die Popularklage, was aber wegen der Vielzahl möglicher Klagen praktisch nicht realisierbar sein dürfte. ${ }^{65}$ Darüber hinaus ist der Schutz und die Weiterentwicklung der Grundrechte einer aus 10 Personen bestehenden Menschenrechtskommission (Art. 115ff) und für das spezielle Grundrecht der Gleichheit der Commission on gender Equality (Art. 119f) anvertraut, die mit weitreichenden Möglichkeiten ausgestattet sind.

Offen geblieben ist hingegen die Frage der Drittwirkung der Grundrechte. Meinungsverschiedenheiten ergeben sich insofern daraus, als das mit der Ausarbeitung des Grundrechtskapitels betraute technische Komitee eine horizontale Wirkung der Grundrechte ausschließen wollte und so unter Ausschluß der Judikative in Art. 7 Abs. 1 formulierte: This Chapter shall bind all legislative and executive organs of state at all levels of government. Auf der anderen Seite bindet aber Art. 4 Abs. 2 alle staatlichen Organe einschließlich der Rechtsprechung und kommt darüber hinaus den Gerichten nach Art. 35 die besondere Aufgabe zu, bei der Auslegung des Grundrechtskapitels die Werte zu fördern, die einer auf Freiheit und Gleichheit basierenden, offenen und demokratischen Gesellschaft zugrunde liegen. Diese Frage wird das Verfassungsgericht, das im Zeitpunkt

Schedule B of the Canadian Constitution Act of 1982.

65

Es gibt bislang keine Abgrenzung zum vorkonstitutionellen Recht, was auch als Systembruch angesehen werden müßte, da die ganze Verfassungsgebung auf der Kontinuität und der Verbindung des Alten mit dem Neuen beruht. 
des Erscheinens dieses Artikels wohl bereits tätig geworden sein wird, abschließend entscheiden müssen.

\section{Die Provinzen}

Wie bereits einleitend erwähnt, ist das Staatsgebiet der Republik Südafrika mit Inkrafttreten der neuen Verfassung in 9 neue Provinzen, die den Ländern im Bund entsprechen, aufgeteilt worden. ${ }^{66}$ Das Territorium jeder Provinz ist größtenteils in Teil 1 des ersten Scheduls definiert. Die dort getroffene Aufteilung richtet sich nach den Magisterial Districts, wie sie der Magistrates Court Act 32 von 1944 zu einer Zeit schuf, als noch keine Homelands aus dem Staatsgebiet ausgegliedert worden waren. Für vierzehn Gebiete, die in Schedule 1, Teil 2 genannt sind, mag sich jedoch die geographische Zuteilung zu einer Provinz auf das Geheiß der in diesen Gebieten lebenden Wähler oder der politischen Partei, die die Mehrheit der dort wohnenden Wähler repräsentiert, nach dem dort näher bestimmten Verfahren verändern. Ansonsten können Provinzgrenzen nur noch durch ein besonderes Gesetz geändert werden, was für seine Gültigkeit nach Art. 61 und 62 der Zustimmung der Nationalversammlung, der diese Provinz vertretenden Senatoren und des Provinzparlaments bedarf.

Eine der Eventualitäten, die eine Veränderung der Provinzgrenzen möglich werden lassen könnte, kann in der Annahme der durch den Volksstaat Council gemachten Vorschläge zur Errichtung und den Grenzen eines durch weiße Bewohner geprägten, sich selbstbestimmenden Volksstaats durch das Parlament sein, da Art. 184B Abs. 3 eine zuvor getroffene Festlegung der Provinzen dem nicht entgegenstehen läßt. Dieser Council, der durch das erste Amendment zur Verfassung in Kapitel XXIA geschaffen wurde, wird aus 20 Parlamentsmitgliedern bestehen, die die Errichtung eines Volksstaats befürworten. Er hat aber lediglich die Macht, Empfehlungen auszusprechen. Im Hinblick auf das 18. Verfassungsprinzip des Schedule 4 bleibt aber zu betonen, daß eine endgültige Errichtung eines Volksstaats der Anerkennung durch die endgültige Verfassung bedarf.

Art. 125 errichtet in jeder Provinz Provinzparlamente, deren Größe von der Electoral Commission in Übereinstimmung mit $\S 10$ Schedule 2 festgelegt wurden. Dabei sollte jedes Provinzparlament wenigstens 30 Sitze und nicht mehr als 100 Sitze - in Abhängigkeit von der Anzahl der repräsentierten Wähler - haben. Insofern bestehen in den Provinzen unterschiedlich große Parlamente, die ebenfalls nach dem Verhältniswahlsystem gewählt wurden. 
Im übrigen gilt hinsichtlich der Zusammensetzung der Provinzregierungen ebenfalls das Modell des Governement of National Unity.

Die Gesetzgebungskompetenzen der Provinzen nun bildeten ein anderes Hauptauseinandersetzungsfeld in den Mehrparteienverhandlungen. Die dabei zunächst verabschiedete sehr schwache Stellung der Provinzen erfuhr jedoch durch die beiden Verfassungsänderungen eine beträchtliche Aufwertung. Nach Art. 160 dürfen die Provinzen eine eigene Provinzverfassung mit Zweidrittel-Mehrheit zu verabschieden, die der Interimsverfassung nicht widersprechen darf, was durch das Verfassungsgericht nachgeprüft wird. Die Gesetzgebungskompetenzen bestimmen sich dann nach Art. 126. Insofern steht den Provinzen grundsätzlich eine Gesetzgebungskompetenz für all diejenigen funktionalen Bereiche zu, die in Schedule 6 der Interimsverfassung bestimmt worden sind.

Unter fünf alternativ vorliegenden Voraussetzungen steht jedoch dem Bundesparlament für die gleichen Materien eine, die Gesetzgebungskompetenz der Provinzen verdrängende, konkurrierende Gesetzgebungskompetenz zu. Aufgrund dieser Durchbrechungen der Gesetzgebungskompetenzen der Provinzen muß schon jetzt befürchtet werden, daß im Laufe der Zeit den Provinzen ein immer geringerer Anteil an der Gesetzgebung zukommt, wie es Entwicklungen überall in der Welt anschaulich belegen können.

Die Finanzausstattung der Provinzen bestimmt sich nach Art. 155 bis 159 und setzt sich aus den beiden Elementen der eigenen Abgabenerhebung und dem Mittelzufluß im vertikalen Finanzausgleich zusammen - das horizontale Element ist noch nicht richtig entwickelt, dürfte aber bei den Empfehlungen der Finanzkommission für die vertikale Mittelzuweisung Berücksichtigung finden.

So steht nach Art. 155 jeder Provinz ein angemessener Anteil am nationalen Finanzaufkommen zu. Dieser setzt sich aus einem Prozentanteil von Einkommenssteuer, Umsatzsteuer und Mineralölsteuer zusammen und wird durch ein von beiden Häusern separat beschlossenes Parlamentsgesetz festgelegt und in Abhängigkeit von den Gegebenheiten in den einzelnen Provinzen an diese verteilt.

Weiterhin steht den Provinzen nach Art. 156 Abs. 1B eine eigene exklusive Abgabenerhebungskompetenz bezüglich Kasinos, Spielgeräten, Lotterien und Wetten zu, und sind sie nach Art. 156 berechtigt, andere Steuern wie Einkommens-, Umsatz- oder andere Verkaufssteuern zu erheben.

Aus der Zusammenschau der nur vermeintlichen Gesetzgebungskompetenzen und der weitgehenden finanziellen Abhängigkeit vom Zentralstaat und seinem Finanzbedarf läßt sich sagen, daß die Provinzen eher dem Zentralstaat untergeordnet sind, als daß sie unabhängige Staatengebilde darstellen. 


\section{Die Rechtsprechung}

Nachdem das 7. Verfassungskapitel ein deutliches Bekenntnis zum Rechtsstaat ablegt und die Unabhängigkeit der Justiz gewährleistet, wird der Gerichtsaufbau unter Erhalt der bestehenden Strukturen um ein Verfassungsgericht und eine Kommission für das Gerichtswesen erweitert.

Das Verfassungsgericht besteht neben einem Präsidenten ${ }^{67}$ aus 10 weiteren Mitgliedern, die durch den Staatspräsidenten für eine einmalige Amtszeit von 7 Jahren ernannt werden. Praktisch erfolgt die Auswahl für vier dieser Richter, die aus den Reihen der Richter am Supreme Court gebildet werden, durch die Empfehlung des Gerichtspräsidenten des Supreme Court und für die restlichen Richter durch die Empfehlungen der Kommission für das Gerichtswesen. ${ }^{68}$

Die Aufgaben des Verfassungsgerichts bestimmen sich aus Art. 98, der im einzelnen die Zuständigkeit des Gerichts festlegt. Hervorzuheben ist, daß damit erstmalig einem südafrikanischen Gericht die Aufgabe übertragen worden ist, Parlamentsgesetze auf ihre Vereinbarkeit mit der Verfassung zu überprüfen und Akte der Gesetzgebung und der Verwaltung an den Grundrechten zu messen. Darüber hinaus kommt dem Verfassungsgericht die wichtige Aufgabe zu, die "endgültige" Verfassung auf ihre Vereinbarkeit mit den Verfassungsprinzipien zu überprüfen. Daraus ergibt sich schon jetzt, daß die konkrete Besetzung des Verfassungsgerichts wesentlichen Einfluß auf die weitere Verfassungsentwicklung in Südafrika nehmen wird. ${ }^{69}$ Gerade die von der Verfassung offen gelassenen Fragen der Abtreibung und der Todesstrafe werden das Gericht wohl bald beschäftigen und mit der Frage konfrontieren, welche Zielsetzung sich das Gericht innerhalb der neuen Verfassung gibt.

Die Kommission für das Gerichtswesen dürfte in diesem Zusammenhang eine kritische Rolle spielen, da sie Besetzungsvorschläge unterbreitet. Diese Kommission setzt sich nach Art. 105 aus 8 Personen der verschiedensten juristischen Bereiche und 9 Personen aus der Politik zusammen.

Zum Präsiuenten ist Arthur Chaskalson ernannt worden, der bereits an der Erarbeitung der Verfassung von Namibia mitgewirkt hatte und als vehementer Verfechter von Grundrechten gilt.

68

69

Bei Entstehung dieses Artikels waren zwar bereits Kandidaten bekannt geworden; da aber die konkrete Ernennung noch nicht erfolgt ist, können auch keine Vermutungen über die tendenzielle Ausrichtung des Verfassungsgerichts angestellt werden. 
8. Die Verfassungsgebende Versammlung und die Erarbeitung und Annahme der nächsten Verfassung

Eine der wichtigsten Aufgaben des neu gewählten Parlaments besteht in der Ausarbeitung und Verabschiedung einer "endgültigen" Verfassung, die mit den in Schedule 4 enthaltenen Verfassungsprinzipien übereinstimmen muß, ${ }^{70}$ so daß über die in diesem Zusammenhang wesentliche Frage der Zusammensetzung des Verfassungsgerichts in der Öffentlichkeit großes Interesse besteht und Zeitungen titelten, daß diese Frage fast schon wichtiger sei als die letzte Wahl. ${ }^{71}$ Die Erarbeitung und Verabschiedung der Verfassung erfolgt durch das Parlament, das in gemeinsamen Sitzungen der Nationalversammlung und des Senat als Verfassungsgebende Versammlung zusammentritt ${ }^{72}$

Jeder dabei entwickelte Verfassungstext muß innerhalb von zwei Jahren ${ }^{73}$, gerechnet ab der ersten Sitzung der Verfassungsgebenden Versammlung, mit wenigstens ZweidrittelMehrheit der Gesamtanzahl der Mitglieder der Verfassungsgebenden Versammlung angenommen werden. Für den Fall, daß diese Zweijahresfrist verstrichen ist, regelt Art. 73 verschieden abgestufte Verfahren, um eine neue Verfassung zu erhalten. Zunächst wird der Verfassungsentwurf einer unabhängigen Kommission, die aus 5 Verfassungsexperten gebildet ist, für 30 Tage vorgelegt, innerhalb derer diese Kommission eine Stellungnahme und Ergänzungen ausarbeitet und dann der Verfassungsgebenden Versammlung zur Verabschiedung vorlegt. Sollte auch dann der Verfassungstext keine Zweidrittel-Mehrheit finden, so ist der dann mit einfacher Mehrheit angenommene Verfassungstext den südafrikanischen Bürgern im Rahmen eines Ref erendums vorzulegen und findet dann eine Annahme, wenn wenigstens $60 \%$ der abgegebenen Stimmen für den Verfassungsentwurf abgegeben werden. Sollte auch dieses keinen Erfolg zeitigen, so löst der Staatspräsident das Parlament auf, um Neuwahlen durchzuführen, so daß das eben beschriebene Verfahren in einer verkürzten Zeitspanne erneut einsetzen kann.

In diesem ganzen Prozeß der Ausarbeitung der nächsten Verfassung bilden die Verfassungsprinzipen eine quasi externe Kontrolle über die Verfassungsgebende Versammlung und verdeutlichen die Macht derjenigen Verhandlungsparteien ${ }^{74}$, die den demokratischen Bekenntnissen einer damals noch zukünftigen ANC-Regierung mißtrauten, insbesondere dadurch, daß diese Verfassungsprinzipien nach Art. 74 nicht ergänzt oder aufgehoben werden können. Daher kommen diese Prinzipien einer verfassungsrechtlichen Garantie in dem Sinn nahe, daß unabhängig von den sich aus den ersten allgemeinen Wahlen erge-

Art. 71 Abs. 1 und 2.

Argus, 20.9.94, S. 12

Art. 68

Dazu und für das nachfolgen beschriebene Verfahren Art. 73 Abs. 1 bis 13

vornehmlich die von der National Party gebildete Regierung und die Inkatha Freedom Party. 
benden Machtverhältnissen bestimmte Strukturen, Verfahren und Interessen Eingang in die nächste Verfassung finden werden.

Eine Entsprechung findet sich in der jüngeren Verfassungsgeschichte nur in den Mindestbedingungen, denen die Verfassung des unabhängigen Namibia zu entsprechen hatte und die durch den Security Council der Vereinten Nationen aufgestellt worden waren. Im Unterschied dazu sind die Verfassungsprinzipien als Resultat der Mehr-Parteien-Verhandlungen selbst auferlegt und verkörpern so einen politischen Vertrag für die Übergabe der Macht von denjenigen, die sie in den Händen hielten, an diejenigen, die bisher von ihr ausgeschlossen waren.

Ihrem Inhalt nach zeichnen sich die Verfassungsprinzipien durch ihre immense Weite aus, die von allgemeinen Festlegungen wie: "The Constitution ... shall provide for the establisment of ... a democratic system of government committed to archieving equality between man and woman and people of all races"75 bis zu ganz speziellen Reglungen wie: " The independence and impartiality of a Puplic Service Commission, a Reserve Bank ... shall be provided for and safeguarded" ${ }^{76}$ und "Every member of the public service shall be entitled to a fair pension"77 reicht. Insofern sind aufgrund dieser Verfassungsprinzipien die wesentlichen Aspekte einer nächsten Verfassung schon vorgezeichnet.

\section{Perspektiven}

Die neue Verfassung verbindet in einzigartiger Weise Altes mit Neuem und versucht einen Interessenausgleich zwischen den verschiedensten Gruppen und insbesondere zwischen den aufgrund der Apartheidspolitik geprägten gesellschaftlichen Verschiedenheiten herzustellen. Dieser Kampf um die Überwindung der Trennung der Menschen voneinander kann aber nur gewonnen werden, wenn Südafrika den Weg des friedlichen Wandels weiterverfolgt, die vielfältigen Wunden der früheren Ungerechtigkeit heilt und mit Hilfe aller eine neue, gerechte Gesellschaft errichtet.

Der dazu eingeschlagene Weg erscheint erfolgversprechend. Gerade durch die den Staatsbediensteten gegebenen Arbeitsplatzgarantien und die zur Zeit stattfindende Integration der politischen Kampf verbände in die reguläre Armee sind deutliche Signale auf ein Zueinander gesetzt worden. Vielfach bedarf es nur einfach des Kennenlernens, was viele Jahre unter Apartheidspolitik nicht möglich war, um so die verschiedenen Mentalitäten zu

75

76

77

Schedule 4 - Principle I.

Schedule 4 - Principle XXIX

Schedule 4 - Principle XXX Nr. 2. 
verstehen. Insofern kann schon die ganz unerhört gestiegene Nachfrage nach Sprachkursen in Xhosa oder Zulu hoffnungsvoll stimmen.

Was nun die Ausarbeitung der neuen Verfassung angeht, so hat die Verfassungsgebende Versammlung ihre Arbeit am 15. August 1994 aufgenommen und einen Leitungsausschuß eingesetzt. Darüber hinaus sollen fünf bis dreißig Themenkomitees gebildet werden, um die Arbeit an der neuen Verfassung zu beschleunigen. Nach ersten Äußerungen soll die Diskussion insbesondere über Fragen des Wahlsystems, das dann auf Wahlkreisen basieren soll, über die Aufnahme von sozio-ökonomischen Grundrechten und der Kommunalverwaltungsstrukturen wieder eröffnet werden. Besondere Bedeutung kommt in diesem Zusammenhang der Absicht zu, den Diskussionsprozeß nicht wie ehemals hinter verschlossenen Türen zu führen, sondern der breiten Mitwirkung und Diskussion in der Öffentlichkeit zu öffnen. ${ }^{78}$

Südafrika als Land der Gegensätze, oder - wie gerne gesagt wird - als eine ganze Welt in einem Land, steht vor großen Herausforderungen. So stehen einer friedlichen Entwicklung nicht nur Probleme wie die Aufspaltung der Gesellschaft in eine 1. und 3. Welt, ein hohes Bevölkerungswachstum, Arbeitslosigkeit, Wohnungslosigkeit und Armut entgegen, sondern auch die erschreckend hohe Rate an Analphabeten, die sich abzeichnende Wasserknappheit und bisher praktizierte Intoleranz. In dieser Situation vollzieht sich ein bislang friedlicher Machtwechsel, der von vorausdenkenden Politikern eingeleitet wurde und immer größere Akzeptanz in der Bevölkerung fand. Gerade das Vertrauen in das Recht ist dabei sehr groß, fast schon zu groß, möchte man meinen.

Angesichts dieser Vielfältigkeit sollte in dem begonnenen Umgestaltungsprozeß die Frage nach der diese Vielschichtigkeit am besten berücksichtigenden Aufgaben- und Machtverteilung zwischen den verschiedenen staatlichen Ebenen nicht zu kurz kommen. Auf Dauer wird gerade die überzeugende und minderheitengerechte Lösung dieser Frage über den friedlichen Bestand und das Wohlergehen Südafrikas entscheiden. process should involve all South Af ricans and not just the powerful". 


\section{ABSTRACTS}

\section{Parliamentary Elections in South Africa}

\section{By Ulf Engel}

Despite of administrative and logistical problems, attempts of both sabotage and fraud, and the prevailing climate of political intolerance and violence the South African people were able to cast their votes in parliamentary elections held on April 26-29, 1994. The decisive phase of transition from apartheid rule to multiparty democracy was, thus, brought to an end. Both the national Independent Election Commission and the international community regarded these elections as "substantially free and fair". On the one hand the success of N. Mandela's ANC - which formed a coalition government with the NP of former President F.W. de Klerk and the rival IFP of M. Buthelezi - allowed the political centre to continue its "negotiated revolution", on the other it gave way to the full reintegration of South Africa in international relations.

This article presents a reconstruction of the final stage of this transitional process. After a short summary of the main developments leading to a negotiated settlement (1989-93), analysis is on 1994, i.e. the implosion of the homeland of Bophuthatswana and continued negotiations with IFP, PAC and the rightwing parties in order to reach an "inclusive settlement". The focus is also on technical and political aspects of election preparations and the security situation in the run-up to the elections. Special emphasis is on the voting and counting process and the assessment of these elections by different election observer missions.

\section{South Africa's new Interim Constitution}

\section{By Hans Friedrich Heese and Thomas H. Böhnke}

The Republic of South Africa undergoes a deep change from Apartheid into a new society based on equality of all races. For the first time South Africa will be a constitutional state with the constitution including a justificable bill of rights being the supreme law of the country.

The new Interim Constitution concludes the structure of the South African state for the next five years. This Constitution is deliberately a transitional instrument, a means 
whereby a democratic-elected legislature can act legally to draw up a „final“ constitution in accordance to the 34 constitutional principles in Schedule 4 of the actual Constitution. This same legislature and the executive drawn from its ranks is entitled by law to govern for a period of five years, even though a final constitution should have been agreed on after only two years.

\section{The Right to Clean Environment: Lessons from India and Tanzania}

\section{By Emmanuel Opoku Awuku}

The need for environmental protection has brought about the international recognition of the right to a clean environment expressed in various international declarations and resolutions. In order to implement the right to a clean environment, it must be reflected in national constitutions and laws of individual states. India and Tanzania serve as examples here for an analysis of the problems in implementing and enforcing the right to a clean environment.

Ratifying international agreements on environmental issues, India included the right to clean environment in ist constitution. It is clearly stated in the Directive Principles. The states of India have to comply with these Directive Principles when making laws. The Directive Principles are, however, not enforceable in court. Various case studies show that the right to a clean environment can be enforced by linking it, for example, with the right to life enshrined in the Indian constitution. The importance of public interest litigation groups is shown.

The constitution of Tanzania does not state the right to a clean environment. It contains, however, some environmental provisions. But these are too general and very difficult to enforce.

Despite the environmental provisions in the constitutions of both India and Tanzania, the laws remain inadequate and the difficulties in enforcing them persist and with it the environmental problems. 